\title{
Consumo diário de refrigerantes, doces e frituras em adolescentes do Nordeste brasileiro
}

\author{
Daily consumption of soft drinks, sweets and fried foods \\ among adolescents in the Northeast of Brazil
}

Roberta de Vargas Zanini ${ }^{1}$

Ludmila Correa Muniz ${ }^{1}$

Bruna Celestino Schneider ${ }^{1}$

Rafael Miranda Tassitano ${ }^{2}$

Wallacy Milton do Nascimento Feitosa ${ }^{3}$

David Alejandro González-Chica ${ }^{4}$

\footnotetext{
${ }^{1}$ Programa de Pós-

Graduação em

Epidemiolgia, Universidade Federal de Pelotas.

Marechal Deodoro 1160/30

Centro. 96.020-220 Pelotas

RS Brasil.

robe.nutri@gmail.com

${ }^{2}$ Departamento de Educação

Física, Universidade Federal

Rural de Pernambuco.

${ }^{3}$ Faculdade ASCES.

${ }^{4}$ Departamento de Nutrição,

Universidade Federal de

Santa Catarina.
}

\begin{abstract}
A school-based cross-sectional study in 2007 evaluated the prevalence and associated factors of daily consumption of soft drinks, sweets and fried foods among adolescents (15 to 20 years of age) in public schools in Caruaru in the state of Pernambuco. To evaluate the factors associated with the daily consumption of the above foods, a multivariate and hierarchical analysis was conducted using Poisson regression, with social and demographic variables at the first hierarchical level, behavioral variables at the second level and $d i-$ etary standards at the third level. Consumption of soft drinks, sweets and fried foods at least once a week was declared by $90.9 \%, 95.4 \%$ and $89.6 \%$ of the adolescents, respectively. The corresponding prevalence of the daily consumption of these items was $30.2 \%, 42 \%$ and $28.3 \%$. The daily consumption of sweets was $21 \%$ higher among girls and $25 \%$ higher among adolescents who ate rice and beans daily. With respect to fried foods, girls mentioned $37 \%$ greater consumption than boys. Adolescents who consumed meat every day admitted a $43 \%$ higher daily consumption of fried foods. The consumption of soft drinks, sweets and fried foods among the adolescents from Caruaru was high and showed a homogeneous consumption standard for most variables analyzed.
\end{abstract}

Key words Food consumption, Soft drinks, Sweets, Adolescents, Cross-sectional study, Dietary record
Resumo Estudo transversal de base escolar que avaliou a prevalência e fatores associados ao consumo diário de refrigerantes, doces e frituras em adolescentes (15-20 anos) da rede pública estadual de Caruaru (PE), em 2007. Para avaliar os fatores associados ao consumo diário de refrigerantes, doces e frituras, realizou-se análise multivariável e hierarquizada, por regressão de Poisson, com variáveis sociodemográficas no primeiro nível hierárquico, comportamentais no segundo e relacionadas ao padrão alimentar no terceiro. O consumo de refrigerantes, doces ou frituras em pelo menos uma vez por semana foi referido por $90,9 \%$, 95,4\% e 89,6\% dos adolescentes, respectivamente. As prevalências correspondentes ao consumo diário destes alimentos foram 30,2\%, 42,0\% e 28,3\%. O consumo diário de doces foi $21 \%$ maior entre as meninas e 25\% maior entre adolescentes que consumiram arroz e feijão diariamente. Em relação às frituras, as meninas referiram um consumo $37 \%$ maior do que os meninos. Adolescentes que consumiram carnes diariamente, apresentaram um consumo diário de frituras $43 \%$ maior. O consumo de refrigerantes, doces e frituras entre os adolescentes caruaruenses mostrou-se elevado e apresentou um padrão de consumo homogêneo para a maioria das variáveis analisadas.

Palavras-chave Consumo alimentar, Refrigerantes, Doces, Adolescentes, Estudo transversal, Registros dietéticos 


\section{Introdução}

O consumo de refrigerantes, gorduras saturadas e alimentos processados tem aumentado consideravelmente nas últimas décadas em vários países ${ }^{1}$. Este aumento no consumo de bebidas com adição de açúcar é preocupante, pois atualmente os refrigerantes são a principal fonte de açúcar simples na dieta de jovens e adultos, sendo responsáveis por aproximadamente $30 \%$ da energia diária de açúcar consumido ${ }^{2}$. Além disso, o consumo frequente de alimentos com alta concentração de energia (gorduras e/ou açúcares) tem sido considerado um fator importante para o aumento observado nas prevalências de sobrepeso e obesidade, assim como no incremento da prevalência de diversas Doenças Crônicas Não Transmissíveis (DCNT) ${ }^{3}$.

Nos últimos trinta anos, no Brasil, foi possível observar uma mudança expressiva no perfil alimentar da populaçãa ${ }^{3,4}$. Resultados das Pesquisas de Orçamentos Familiares (POF), desenvolvidas pelo Instituto Brasileiro de Geografia e Estatística (IBGE) no período entre 1974-2009, revelaram mudanças importantes ocorridas na alimentação brasileira ${ }^{5}$. Neste período, a participação relativa dos lipídios na dieta aumentou de $25,7 \%$ para $30,5 \%$, sendo que na última avaliação (2002-2003) o consumo de ácidos graxos saturados atingiu o limite máximo recomendado pelos referenciais da alimentação saudável $(9,6 \%)^{6}$. Além disso, nas últimas três décadas a participação do açúcar industrializado (sacarose) na dieta ultrapassou o limite de $10 \%$ recomendado pela Organização Mundial da Saúde ${ }^{7}$. Nesse mesmo período houve também aumento na disponibilidade de embutidos (cerca de 300\%), biscoitos, açúcar e refrigerantes (400\%), o que indica uma forte tendência de aumento no consumo de produtos industrializados ${ }^{3}$.

Diante das mudanças ocorridas nos padrões de alimentação e do aumento da prevalência de obesidade e DCNT, o Ministério da Saúde publicou em 2006 o Guia Alimentar para a População Brasileira, o qual recomenda a redução do consumo de bebidas e alimentos processados com alta concentração de açúcar e/ou gordura, e orienta para que não ultrapasse a quantidade de uma porção diária ${ }^{8}$. Nos últimos anos, o aumento na prevalência de excesso de peso foi evidenciado em todos os grupos etários ${ }^{6}$, no entanto, esta condição durante a adolescência torna-se importante, tendo em vista que, neste período, novos hábitos alimentares estão sendo adquiridos e consolidados. Estas escolhas alimentares podem ser influenciadas por diversos fatores econômicos, culturais, sociais e ambientais, que por sua vez, podem alterar os hábitos do adolescente em nível individual, familiar e/ou escolar9.

A dieta dos adolescentes tem sido caracterizada nos últimos anos pela preferência por alimentos ricos em gordura e de elevado conteúdo calórico, incluindo os alimentos prontos para o consumo ou fast foods, frituras e bebidas com adição de açúcar ${ }^{10,11}$. No Brasil, são escassos os estudos que avaliam o consumo desses alimentos entre adolescentes, especialmente nas regiões norte e nordeste do Brasil. Nestas regiões foram localizados apenas três estudos de prevalência realizados nos estados de Fortaleza ${ }^{12}$, Paraíb $^{13} \mathrm{e}$ Bahia $^{14}$, os quais revelaram uma frequência de consumo de refrigerantes, doces e frituras entre $20-40 \%$. No entanto, são limitadas as evidências que estes estudos fornecem sobre os possíveis fatores associados ao consumo destes alimentos, os quais seriam fundamentais para o direcionamento das políticas de saúde que visam à redução do seu consumo.

Diante do exposto, o objetivo deste trabalho foi descrever a frequência de consumo de refrigerantes, doces e frituras entre adolescentes estudantes da rede pública de ensino do município de Caruaru (PE), e avaliar os fatores associados, com ênfase nos sociodemográficos, estilo de vida, estado nutricional e consumo de outros alimentos.

\section{Metodologia}

Estudo transversal de base escolar realizado em Caruaru (PE). A cidade está localizada na região agreste do estado, distante $120 \mathrm{~km}$ de Recife, com uma população estimada de 289.086 habitantes em 2007. A população alvo deste estudo foram todos os alunos do ensino médio da rede pública estadual, com idades entre 15 e 20 anos, distribuídos nas 15 escolas do município.

O cálculo para o tamanho da amostra foi realizado com o auxílio do programa SampleXS, distribuído pela OMS para apoiar o planejamento amostral em estudos transversais. Para este cálculo foi utilizada uma prevalência estimada de $50 \%$ na tentativa de maximizar o número de indivíduos a serem incluídos no estudo, considerando a escassez de informação sobre o assunto para esta população. Foram incluídos ainda os seguintes parâmetros: intervalo de confiança de $95 \%$, erro máximo tolerável de cinco pontos percentuais e efeito de delineamento amostral de 1,5. Dessa forma, o tamanho amostral necessário foi 
de 520 adolescentes. Posteriormente, foram acrescidos $20 \%$ para eventuais perdas e recusas e controle para fatores de confusão, sendo necessária uma amostra final de 649 estudantes.

O processo de amostragem foi realizado em duplo estágio por conglomerados. Todos os sorteios foram realizados mediante o programa randomizer, que forneceu números aleatórios. A unidade amostral primária foi a escola, sendo consideradas elegíveis para entrar no estudo todas aquelas da rede estadual que ofereciam ensino médio. A seleção aleatória considerou a proporção de escolas e de estudantes em cada microrregião do município, bem como o porte da mesma. À época do estudo, Caruaru contava com 15 escolas públicas estaduais: quatro de grande porte (mais de 500 alunos), sete de tamanho médio (entre 200 e 499 alunos) e quatro de pequeno porte (menos que 200 alunos). Dessa forma, foram selecionadas oito escolas estaduais (duas escolas de grande porte, quatro de médio porte e duas pequenas), garantindo que pelo menos 50\% das escolas de cada tamanho fossem selecionadas.

No segundo estágio, a unidade amostral foi a turma, considerando-se o turno escolar (diurno ou noturno). A amostragem aleatória considerou como critério de estratificação a densidade de turmas em cada escola sorteada e a quantidade de estudantes matriculados em cada turno. Nesta fase foram selecionadas aleatoriamente duas turmas em cada uma das oito escolas incluídas na primeira fase (16 turmas no total). De acordo com informações fornecidas pela Secretaria da Educação e Cultura (SEDUC) do Estado de Pernambuco, referentes ao censo escolar de 2007, havia em média, 41 alunos matriculados em cada turma de ensino médio das escolas estaduais do município.

A coleta dos dados ocorreu durante o mês de outubro do ano de 2007, sendo selecionados todos os alunos presentes em sala de aula no dia da entrevista. Previamente os diretores foram contatados para o envio dos termos de consentimento e agendamento da visita. $\mathrm{Na}$ escola, os questionários eram distribuídos para os alunos e, em seguida, o pesquisador realizava uma leitura de cada pergunta, para que, posteriormente os alunos respondessem às questões (entrevista coletiva/autoaplicada). Em caso de dúvida, a leitura era interrompida, e as dúvidas, esclarecidas, conforme combinado previamente em treinamento interno.

Para a coleta das informações utilizou-se o questionário intitulado "Comportamentos de ris- co em adolescentes Catarinenses" (COMCAP), previamente validado em estudantes da rede de ensino brasileira ${ }^{15}$. Adicionalmente, realizou-se pré-teste das questões em 60 estudantes de 15 a 20 anos de idade, matriculados em duas escolas caruaruenses não selecionadas para participar do estudo. O questionário abrange informações relacionadas ao estilo de vida do adolescente, sendo composto por uma sessão de informações gerais (sociodemográficas e relacionadas ao trabalho) e cinco relacionadas à saúde (hábitos alimentares, atividade física, comportamentos de risco, comportamentos preventivos e percepção de saúde).

Como variáveis dependentes foram consideradas as frequências de consumo de refrigerantes, doces e frituras. Para tanto, as informações foram coletadas através de três perguntas: "Quantas vezes você toma refrigerante?"; "Quantas vezes você come salgadinhos fritos (batata frita, chips, coxinhas, pastéis e outros)?" e "Quantas vezes você come docinhos, tortas, chocolate, biscoitos, bolachas ou balas?". As opções de resposta foram as mesmas para as três perguntas: "Nenhuma vez"; "1 a 3 vezes por semana"; "4 a 6 vezes por semana"; "Todos os dias/1 vez por dia"; "Todos os dias/2 vezes por dia" e "Todos os dias/ 3 vezes por dia”.

Para as análises, as variáveis que compõem o desfecho em estudo foram agrupadas em "consumo diário" e "menor/igual a 6 vezes por semana”. As variáveis independentes avaliadas foram: sexo (masculino/feminino); idade (15-17/18-20 anos); número de moradores no domicílio (1-4 $\geq 5)$; renda familiar em reais $(\leq 1000 / \geq 1001)$; zona de residência (urbana/rural); hábito de fumar atual independente do número de cigarros

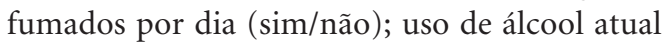
independente da frequência e da quantidade ingerida ( $\operatorname{sim} /$ não); prática de atividade física (insuficientemente ativo: $\operatorname{sim} /$ não); satisfação com o peso (insatisfeito/satisfeito); autopercepção de saúde (excelente/boa/regular-ruim); excesso de peso ( $\operatorname{sim} /$ não); consumo de 3 refeições/dia (sempre/às vezes-nunca); e variáveis relativas à alimentação: consumo diário de frutas e legumes ( $\operatorname{sim} /$ não); de carnes em geral ( $\operatorname{sim} /$ não) e de feijão com arroz (sim/não). As informações sobre atividade física foram obtidas a partir da versão curta do Questionário Internacional de Atividade Física/IPAQ ${ }^{16}$. Foram considerados insuficientemente ativos os indivíduos que informaram praticar menos de 150 minutos de atividade física por semana.

A técnica para a coleta das medidas antropométricas (peso e altura) seguiu as recomenda- 
ções de Lohman et al. ${ }^{17}$ A medida do peso corporal foi obtida utilizando uma balança eletrônica da marca Plenna (modelo Sport), com capacidade máxima de $150 \mathrm{Kg}$ e precisão de 100 gramas. A estatura foi aferida com auxílio de um estadiômetro da marca Plenna (modelo 206), com altura máxima de 2,2 metros e precisão de $5 \mathrm{~cm}$. Para classificação do estado nutricional dos indivíduos utilizou-se os critérios da $\mathrm{OMS}^{18,19}$, com base no Índice de Massa Corporal (IMC) obtido pela divisão do peso (em quilogramas) pela altura (em metros) ao quadrado. Foram considerados com excesso de peso os adolescentes que apresentaram IMC/idade $\geq+1,0$ escores $\mathrm{z}$ na curva de referência e, para aqueles indivíduos com 20 anos de idade, foi usado como ponto de corte o IMC $\geq 25 \mathrm{Kg} / \mathrm{m} 2$.

Os dados foram digitados no programa Epi Data (versão 3.1). A análise foi realizada com o pacote estatístico Stata (versão 11.0). O efeito de amostragem por conglomerados foi considerado em todas as análises, utilizando-se o conjunto de comandos "survey" (svy). Nas análises brutas e ajustadas foram estimadas razões de prevalência (RP) com intervalos de confiança (IC95\%), calculados por meio de regressão de Poisson ${ }^{20}$. A significância estatística de cada variável no modelo foi avaliada através dos testes de Wald de heterogeneidade ou de tendência linear. Para a análise multivariável elaborou-se um modelo hierárquico, conceitual, no qual as variáveis sociodemográficas situavam-se no primeiro nível, as comportamentais no segundo e aquelas relacionadas ao consumo alimentar no mais proximal ao desfecho. A seleção de variáveis para ajuste considerou o procedimento de seleção para trás $(b a-$ ckward) por níveis. De acordo com este método, todas as variáveis socioeconômicas ( $1^{\circ}$ nível) foram ajustadas entre si, independente do nível de significância da análise bruta, sendo mantidas no modelo unicamente aquelas que apresentaram valor $\mathrm{p}<0,20$ na análise ajustada. Posteriormente, foram incluídas todas as variáveis comportamentais ( $2^{\circ}$ nível), as quais foram ajustadas para as do primeiro nível que permaneceram no modelo, e para as do segundo com $p<0,20$. Finalmente, as variáveis de consumo alimentar foram incluídas nas análises, sendo ajustadas para as variáveis dos dois níveis anteriores que permaneceram no modelo e para as do terceiro nível com $\mathrm{p}<0,20$. As variáveis com $\mathrm{p}<0,05$ foram consideradas como fatores associados aos desfechos.

Este estudo foi aprovado pelo Comitê de Ética em Pesquisa com seres humanos da Associação Caruaruense de Ensino Superior (ASCES).

\section{Resultados}

Um total de 8 escolas e 16 turmas participaram da pesquisa. Do total de estudantes que estavam presentes nos dias de coleta $(n=624), 21$ recusaram-se a participar e em 3 casos não foram obtidas informações sobre consumo alimentar, resultando em $3,8 \%$ de perdas. No final foram obtidas informações de 600 adolescentes com idades entre 15 e 20 anos. A média de idade foi de $17,4$ anos (DP $\pm 1,5)$ e a distribuição segundo o gênero mostrou predominância do sexo feminino $(62,3 \%)$. O consumo geral de refrigerantes, doces ou frituras em pelo menos uma vez na semana mostrou-se elevado, sendo referido por $90,9 \%, 95,4 \%$ e $89,6 \%$ dos adolescentes, respectivamente. Cabe destacar que o consumo diário de cada um desses alimentos também foi elevado, sendo que os doces foram os mais referidos $(42,0 \%)$, seguidos pelos refrigerantes $(30,2 \%)$ e as frituras $(28,3 \%)$. O consumo diário destes três alimentos juntos foi referido por $10 \%$ dos adolescentes, enquanto que $18 \%$ referiram consumir apenas dois destes produtos todos os dias e cerca de $40 \%$ dos adolescentes referiram não consumir nenhum destes alimentos diariamente (Figura 1). A Tabela 1 apresenta a distribuição da frequência de consumo de refrigerantes, doces e frituras, estratificada por sexo. Não houve diferença estatisticamente significativa entre os sexos para o consumo desses alimentos, no entanto, ao considerar o consumo de uma ou mais vezes ao dia para doces e para frituras, a prevalência de consumo mostrou-se maior entre as meninas em relação aos meninos.

A Tabela 2 apresenta as análises bruta e ajustada do consumo diário de refrigerantes de acordo com as variáveis estudadas. Os resultados não apresentaram associação estatisticamente significativa com o desfecho antes e após ajuste para confusão. No entanto, observa-se que o consumo diário de refrigerantes foi, aproximadamente, $25 \%$ menor entre adolescentes com excesso de peso, $35 \%$ menor entre adolescentes que não consomem carnes diariamente e $40 \%$ menor entre os fumantes, em relação às respectivas categorias de referência.

Na Tabela 3 observam-se as Razões de Prevalência (RP) bruta e ajustada do consumo diário de doces de acordo com as características avaliadas. Similar ao ocorrido para o consumo de refrigerantes, muitas das exposições não apresentaram associação significativa com o consumo diário de doces, mesmo após ajuste para possíveis fatores de confusão. No entanto, o con- 


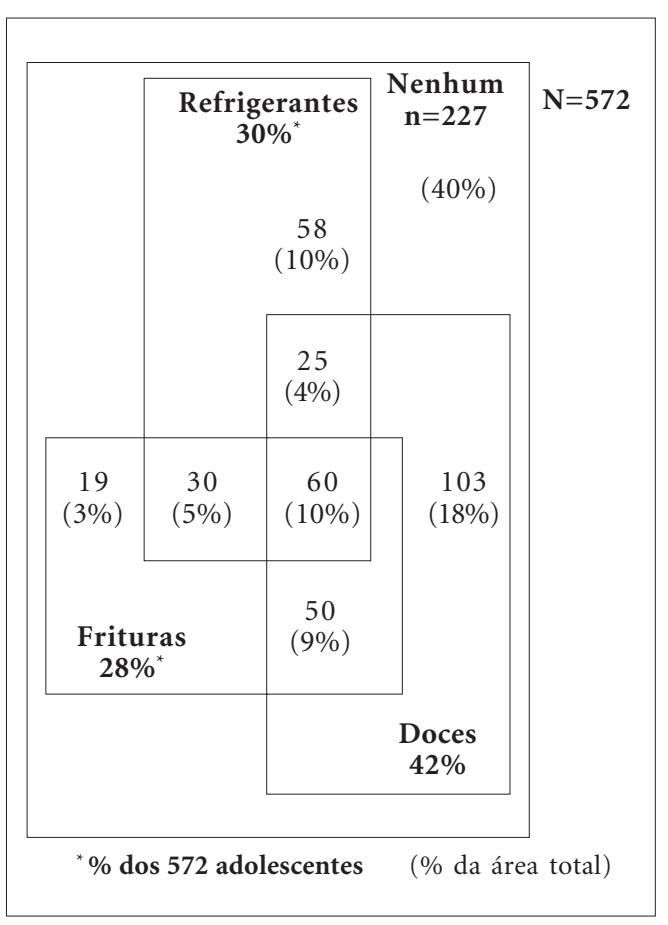

Figura 1. Diagrama de Venn das prevalências de consumo diário de refrigerantes, doces e frituras em adolescentes de Caruaru (PE), 2007. sumo diário de doces foi $21 \%$ maior entre as meninas em relação aos meninos e $25 \%$ menor entre indivíduos que não consomem diariamente a combinação alimentar arroz e feijão, antes e após ajuste para fatores de confusão, sendo estas diferenças estatisticamente significativas. A variável excesso de peso apresentou associação limiar com o desfecho em ambas as análises, sendo a prevalência de consumo de doces 31\% maior entre indivíduos com excesso de peso em relação àqueles com peso normal.

A Tabela 4 apresenta os resultados do consumo diário de frituras. Estudantes que consumiram carnes diariamente referiram um consumo de frituras 55\% maior em relação àqueles que não consumiram. As demais variáveis estudadas não apresentaram associação estatisticamente significativa com o desfecho, no entanto, cabe mencionar que adolescentes que realizaram três refeições por dia apresentaram consumo diário de gorduras $25 \%$ maior do que a categoria de referência, e as meninas um consumo de frituras $27 \%$ maior em relação aos meninos.

Tabela 1. Frequência de consumo de refrigerantes, doces e frituras segundo o sexo. Caruaru (PE), 2007.

\begin{tabular}{|c|c|c|c|}
\hline \multirow[b]{2}{*}{ Frequência de consumo } & \multicolumn{2}{|c|}{ Sexo } & \multirow[b]{2}{*}{ Valor- $\mathbf{p}^{*}$} \\
\hline & $\begin{array}{c}\text { Masculino }(\mathbf{n}=226) \\
\%(\text { IC95\%) }\end{array}$ & $\begin{array}{c}\text { Feminino }(\mathbf{n}=374) \\
\%(I C 95 \%)\end{array}$ & \\
\hline Refrigerantes & & & 0,8 \\
\hline Nunca & $9,1(5,9-12,3)$ & $8,5(4,4-12,7)$ & \\
\hline 1-3 vezes/semana & $43,4(37,3-49,5)$ & $42,3(37,2-47,4)$ & \\
\hline 4-6 vezes/semana & $19,6(14,8-24,5)$ & $17,6(13,7-21,5)$ & \\
\hline $1 \mathrm{vez} / \mathrm{dia}$ & $12,3(4,6-20,0)$ & $12,6(6,1-19,1)$ & \\
\hline $2-3$ vezes/dia & $15,5(11,1-20,0)$ & $19,0(16,4-21,5)$ & \\
\hline Doces & & & 0,1 \\
\hline Nunca & $3,6(1,8-5,4)$ & $5,1(2,0-8,3)$ & \\
\hline $1-3$ vezes/semana & $37,8(29,6-46,1)$ & $31,7(28,4-35,0)$ & \\
\hline 4-6 vezes/semana & $22,5(17,1-28,0)$ & $17,6(11,9-23,3)$ & \\
\hline $1 \mathrm{vez} / \mathrm{dia}$ & $18,5(13,3-23,7)$ & $21,4(18,6-24,2)$ & \\
\hline $2-3$ vezes/dia & $17,6(10,9-24,3)$ & $24,1(20,5-27,8)$ & \\
\hline Frituras & & & 0,2 \\
\hline Nunca & $12,8(9,0-16,7)$ & $9,0(5,5-12,6)$ & \\
\hline 1-3 vezes/semana & $44,5(30,9-58,1)$ & $42,6(37,8-47,4)$ & \\
\hline 4-6 vezes/semana & $19,7(16,2-23,3)$ & $16,9(11,9-22,0)$ & \\
\hline $1 \mathrm{vez} / \mathrm{dia}$ & $12,8(5,9-19,8)$ & $15,0(9,6-20,5)$ & \\
\hline $2-3$ vezes/dia & $10,1(6,4-13,8)$ & $16,4(11,6-21,2)$ & \\
\hline
\end{tabular}

* Teste qui-quadrado de heterogeneidade. ${ }^{* *} \mathrm{O}$ máximo de informações perdidas foi $17(2,8 \%)$ para a variável consumo diário de refrigerantes. 
Tabela 2. Análise bruta e ajustada da associação entre consumo diário de refrigerantes e as variáveis independentes estudadas ( $\mathrm{N}=600)$. Caruaru (PE), 2007.

\begin{tabular}{|c|c|c|c|c|c|c|c|c|}
\hline \multirow{2}{*}{ Variáveis } & \multirow{2}{*}{$\mathbf{N}$} & \multirow{2}{*}{ Prev. } & \multicolumn{3}{|c|}{ Análise bruta } & \multicolumn{3}{|c|}{ Análise ajustada } \\
\hline & & & $\mathbf{R P}$ & IC95\% & $\mathbf{p}^{*}$ & $\mathbf{R P}$ & IC95\% & p \\
\hline Sexo & & & & & 0,4 & & & 0,3 \\
\hline Feminino & 364 & 31,6 & 1,00 & - & & 1,00 & - & \\
\hline Masculino & 219 & 27,9 & 0,88 & $0,66-1,18$ & & 0,88 & $0,66-1,17$ & \\
\hline Idade & & & & & 0,6 & & & 0,4 \\
\hline $15-17$ & 325 & 30,8 & 1,00 & - & & 1,00 & - & \\
\hline $18-20$ & 258 & 29,5 & 0,96 & $0,79-1,16$ & & 0,92 & $0,75-1,14$ & \\
\hline Número de moradores & & & & & 0,4 & & & 0,4 \\
\hline $1-4$ & 321 & 30,8 & 1,12 & $0,84-1,51$ & & 1,13 & $0,84-1,51$ & \\
\hline$\geq 5$ & 215 & 27,4 & 1,00 & - & & 1,00 & - & \\
\hline Renda familiar (R\$) & & & & & 0,3 & & & 0,3 \\
\hline$\leq 1000$ & 429 & 29,1 & 1,00 & - & & 1,00 & - & \\
\hline$\geq 1001$ & 154 & 33,1 & 1,14 & $0,89-1,45$ & & 1,14 & $0,89-1,47$ & \\
\hline Zona de residência & & & & & 0,6 & & & 0,5 \\
\hline Rural & 69 & 33,3 & 1,00 & - & & 1,00 & - & \\
\hline Urbana & 514 & 29,8 & 0,89 & $0,59-1,35$ & & 0,88 & $0,58-1,33$ & \\
\hline Tabagismo & & & & & 0,1 & & & 0,2 \\
\hline Não & 564 & 30,7 & 1,00 & - & & 1,00 & - & \\
\hline Sim & 19 & 15,8 & 0,51 & $0,23-1,16$ & & 0,57 & $0,25-1,27$ & \\
\hline Consumo de bebida alcoólica & & & & & 0,2 & & & 0,4 \\
\hline Não & 312 & 32,1 & 1,00 & - & & 1,00 & - & \\
\hline Sim & 271 & 28,0 & 0,87 & $0,71-1,07$ & & 0,93 & $0,76-1,13$ & \\
\hline Atividade física & & & & & 0,6 & & & 0,6 \\
\hline Sedentário & 240 & 29,2 & 0,96 & $0,81-1,14$ & & 0,96 & $0,80-1,16$ & \\
\hline Ativo & 336 & 30,4 & 1,00 & - & & 1,00 & - & \\
\hline Satisfação com o peso & & & & & 0,7 & & & 0,7 \\
\hline Insatisfeito & 352 & 29,0 & 0,93 & $0,63-1,38$ & & 0,92 & $0,57-1,48$ & \\
\hline Satisfeito & 225 & 31,1 & 1,00 & - & & 1,00 & - & \\
\hline Autopercepção de saúde & & & & & 0,2 & & & 0,3 \\
\hline Excelente & 180 & 33,3 & 1,00 & - & & 1,00 & - & \\
\hline Boa & 269 & 26,8 & 0,80 & $0,55-1,17$ & & 0,81 & $0,55-1,21$ & \\
\hline Regular/ruim & 125 & 33,6 & 1,01 & $0,79-1,29$ & & 1,00 & $0,78-1,29$ & \\
\hline Excesso de Peso & & & & & 0,05 & & & 0,09 \\
\hline Não & 472 & 31,8 & 1,00 & - & & 1,00 & - & \\
\hline Sim & 88 & 23,9 & 0,75 & $0,57-0,99$ & & 0,77 & $0,56-1,04$ & \\
\hline Realiza três refeições/dia & & & & & 0,7 & & & 0,9 \\
\hline Nunca / às vezes & 180 & 29,4 & 0,97 & $0,81-1,16$ & & 1,01 & $0,87-1,18$ & \\
\hline Sempre & 399 & 30,3 & 1,00 & - & & 1,00 & - & \\
\hline Consumo diário de frutas/legumes & & & & & 0,3 & & & 0,4 \\
\hline Não & 75 & 34,7 & 0,85 & $0,61-1,18$ & & 0,87 & $0,61-1,23$ & \\
\hline Sim & 491 & 29,5 & 1,00 & - & & 1,00 & - & \\
\hline Consumo diário de carnes & & & & & 0,06 & & & 0,08 \\
\hline Não & 281 & 23,5 & 0,63 & $0,39-1,03$ & & 0,64 & $0,39-1,06$ & \\
\hline Sim & 296 & 37,2 & 1,00 & - & & 1,00 & - & \\
\hline Consumo diário de arroz e feijão & & & & & 0,6 & & & 0,4 \\
\hline Não & 101 & 28,7 & 0,94 & $0,70-1,26$ & & 1,12 & $0,83-1,50$ & \\
\hline Sim & 477 & 30,6 & 1,00 & - & & 1,00 & - & \\
\hline
\end{tabular}

* Teste de Wald.

\section{Discussão}

A comparação de nossos resultados com outros estudos nacionais apresentam duas possíveis li- mitações. Em primeiro lugar, estudos envolvendo as práticas alimentares de adolescentes brasileiros são escassos na literatura científica, principalmente aqueles relacionados ao consumo de 
Tabela 3. Análise bruta e ajustada da associação entre consumo diário de doces e as variáveis independentes estudadas $(\mathrm{N}=600)$. Caruaru-PE, 2007.

\begin{tabular}{|c|c|c|c|c|c|c|c|c|}
\hline \multirow{2}{*}{ Variáveis } & \multirow{2}{*}{$\mathbf{N}$} & \multirow{2}{*}{ Prev. } & \multicolumn{3}{|c|}{ Análise bruta } & \multicolumn{3}{|c|}{ Análise ajustada } \\
\hline & & & $\mathbf{R P}$ & IC95\% & $\mathbf{p}^{*}$ & $\mathbf{R P}$ & IC95\% & $\mathbf{p}$ \\
\hline Sexo & & & & & 0,008 & & & 0,008 \\
\hline Feminino & 369 & 45,5 & 1,00 & - & & 1,00 & - & \\
\hline Masculino & 222 & 36,0 & 0,79 & $0,67-0,93$ & & 0,79 & $0,68-0,93$ & \\
\hline Idade & & & & & 0,4 & & & 0,3 \\
\hline $15-17$ & 327 & 41,0 & 1,00 & - & & 1,00 & - & \\
\hline $18-20$ & 264 & 43,2 & 1,05 & $0,92-1,21$ & & 1,07 & $0,93-1,23$ & \\
\hline Número de moradores & & & & & 0,9 & & & 0,9 \\
\hline $1-4$ & 327 & 42,2 & 0,99 & $0,84-1,17$ & & 0,99 & $0,83-1,17$ & \\
\hline$\geq 5$ & 214 & 42,5 & 1,00 & - & & 1,00 & - & \\
\hline Renda familiar ( $\mathrm{R} \$$ ) & & & & & 0,4 & & & 0,9 \\
\hline$\leq 1000$ & 434 & 41,2 & 1,00 & - & & 1,00 & - & \\
\hline$\geq 1001$ & 157 & 44,0 & 1,07 & $0,92-1,24$ & & 1,01 & $0,79-1,29$ & \\
\hline Zona de residência & & & & & 0,4 & & & 0,4 \\
\hline Rural & 70 & 38,6 & 1,00 & - & & 1,00 & - & \\
\hline Urbana & 521 & 42,4 & 1,10 & $0,88-1,38$ & & 1,10 & $0,88-1,37$ & \\
\hline Tabagismo & & & & & 1,0 & & & 1,0 \\
\hline Não & 572 & 42,0 & 1,00 & - & & 1,00 & - & \\
\hline Sim & 19 & 42,1 & 1,00 & $0,59-1,70$ & & 0,99 & $0,52-1,90$ & \\
\hline Consumo de bebida alcoólica & & & & & 0,6 & & & 0,7 \\
\hline Não & 319 & 41,1 & 1,00 & - & & 1,00 & - & \\
\hline Sim & 272 & 43.0 & 1,05 & $0,85-1,30$ & & 1,06 & $0,79-1,43$ & \\
\hline Atividade física & & & & & 0,9 & & & 0,3 \\
\hline Sedentário & 244 & 41,8 & 0,99 & $0,83-1,19$ & & 0,91 & $0,74-1,11$ & \\
\hline Ativo & 340 & 42,1 & 1,00 & - & & 1,00 & - & \\
\hline Satisfação com o peso & & & & & 0,3 & & & 0,3 \\
\hline Insatisfeito & 360 & 43,1 & 1,09 & $0,93-1,27$ & & 1,09 & $0,91-1,30$ & \\
\hline Satisfeito & 225 & 39,6 & 1,00 & - & & 1,00 & - & \\
\hline Autopercepção de saúde & & & & & 0,6 & & & 0,7 \\
\hline Excelente & 182 & 40,7 & 1,00 & - & & 1,00 & - & \\
\hline Boa & 273 & 41,0 & 1,01 & $0,78-1,31$ & & 1,00 & $0,74-1,35$ & \\
\hline Regular/ruim & 127 & 46,5 & 1,14 & $0,78-1,68$ & & 1,11 & $0,73-1,69$ & \\
\hline Excesso de Peso & & & & & 0,05 & & & 0,05 \\
\hline Não & 480 & 40,0 & 1,00 & - & & 1,00 & - & \\
\hline Sim & 88 & 52,3 & 1,31 & $1,00-1,70$ & & 1,31 & $1,00-1,71$ & \\
\hline Realiza três refeições/dia & & & & & 0,7 & & & 0,9 \\
\hline Nunca / às vezes & 182 & 43,4 & 1,05 & $0,82-1,36$ & & 1,01 & $0,78-1,30$ & \\
\hline Sempre & 405 & 41,2 & 1,00 & - & & 1,00 & - & \\
\hline Consumo diário de frutas/legumes & & & & & 0,3 & & & 0,2 \\
\hline Não & 76 & 48,7 & 0,86 & $0,64-1,15$ & & 0,86 & $0,65-1,13$ & \\
\hline Sim & 498 & 41,8 & 1,00 & - & & 1,00 & - & \\
\hline Consumo diário de carnes & & & & & 0,5 & & & 0,8 \\
\hline Não & 285 & 40,0 & 0,92 & $0,68-1,23$ & & 0,96 & $0,70-1,32$ & \\
\hline Sim & 302 & 43,7 & 1,00 & - & & 1,00 & - & \\
\hline Consumo diário de arroz e feijão & & & & & 0,003 & & & 0,001 \\
\hline Não & 104 & 33,7 & 0,77 & $0,67-0,89$ & & 0,75 & $0,66-0,85$ & \\
\hline Sim & 483 & 43,7 & 1,00 & - & & 1,00 & - & \\
\hline
\end{tabular}

${ }^{*}$ Teste de Wald.

alimentos industrializados, ricos em açúcares e/ ou gorduras. Após uma revisão bibliográfica, foram localizados onze artigos brasileiros que avaliaram o consumo destes alimentos entre adoles- centes. Destes, três foram realizados em São Pau$10^{21-23}$, quatro na Região Sul ${ }^{24-27}$, um no Rio de Janeiro $^{28}$ e outros três foram conduzidos na Região Nordeste, especificamente nos estados da 
Tabela 4. Análise bruta e ajustada da associação entre consumo diário de frituras e as variáveis independentes estudadas ( $\mathrm{N}=600)$. Caruaru (PE), 2007.

\begin{tabular}{|c|c|c|c|c|c|c|c|c|}
\hline \multirow{2}{*}{ Variáveis } & \multirow{2}{*}{$\mathrm{N}$} & \multirow{2}{*}{ Prev. } & \multicolumn{3}{|c|}{ Análise bruta } & \multicolumn{3}{|c|}{ Análise ajustada } \\
\hline & & & $\mathbf{R P}$ & IC95\% & $\mathbf{p}^{*}$ & $\mathbf{R P}$ & IC95\% & $\mathbf{p}$ \\
\hline Sexo & & & & & 0,2 & & & 0,2 \\
\hline Feminino & 366 & 31,4 & 1,00 & - & & 1,00 & - & \\
\hline Masculino & 218 & 22,9 & 0,73 & $0,45-1,17$ & & 0,73 & $0,45-1,18$ & \\
\hline Idade & & & & & 0,2 & & & 0,2 \\
\hline $15-17$ & 326 & 27,0 & 1,00 & - & & 1,00 & - & \\
\hline $18-20$ & 258 & 29,8 & 1,11 & $0,93-1,32$ & & 1,12 & $0,93-1,34$ & \\
\hline Número de moradores & & & & & 0,4 & & & 0,5 \\
\hline $1-4$ & 322 & 29,5 & 1,07 & $0,91-1,26$ & & 1,06 & $0,90-1,25$ & \\
\hline$\geq 5$ & 214 & 27,6 & 1,00 & - & & 1,00 & - & \\
\hline Renda familiar (R\$) & & & & & 0,7 & & & 0,5 \\
\hline$\leq 1000$ & 428 & 28,0 & 1,00 & - & & 1,00 & - & \\
\hline$\geq 1001$ & 156 & 28,9 & 1,03 & $0,87-1,22$ & & 1,09 & $0,85-1,41$ & \\
\hline Zona de residência & & & & & 0,9 & & & 0,5 \\
\hline Rural & 68 & 27,9 & 1,00 & - & & 1,00 & - & \\
\hline Urbana & 516 & 28,3 & 1,01 & $0,72-1,42$ & & 1,10 & $0,81-1,49$ & \\
\hline Tabagismo & & & & & 0,5 & & & 0,7 \\
\hline Não & 565 & 28,5 & 1,00 & - & & 1,00 & - & \\
\hline Sim & 19 & 21,1 & 0,74 & $0,25-2,19$ & & 0,84 & $0,30-2,33$ & \\
\hline Consumo de bebida alcoólica & & & & & 0,3 & & & 0,2 \\
\hline Não & 314 & 26,4 & 1,00 & - & & 1,00 & - & \\
\hline $\operatorname{Sim}$ & 270 & 30,4 & 1,15 & $0,84-1,57$ & & 1,24 & $0,90-1,70$ & \\
\hline Atividade física & & & & & 0,5 & & & 0,7 \\
\hline Sedentário & 242 & 30,2 & 1,12 & $0,79-1,60$ & & 1,06 & $0,81-1,38$ & \\
\hline Ativo & 335 & 26,9 & 1,00 & - & & 1,00 & - & \\
\hline Satisfação com o peso & & & & & 0,3 & & & 0,4 \\
\hline Insatisfeito & 358 & 25,4 & 0,81 & $0,54-1,21$ & & 0,84 & $0,56-1,26$ & \\
\hline Satisfeito & 220 & 31,4 & 1,00 & - & & 1,00 & - & \\
\hline Autopercepção de saúde & & & & & 1,0 & & & 0,7 \\
\hline Excelente & 180 & 27,8 & 1,00 & - & & 1,00 & - & \\
\hline Boa & 270 & 28,5 & 1,03 & $0,66-1,59$ & & 0,98 & $0,64-1,48$ & \\
\hline Regular/ruim & 125 & 28,8 & 1,04 & $0,61-1,75$ & & 0,87 & $0,54-1,40$ & \\
\hline Excesso de Peso & & & & & 0,3 & & & 0,2 \\
\hline Não & 473 & 29,0 & 1,00 & - & & 1,00 & - & \\
\hline Sim & 88 & 26,1 & 0,90 & $0,73-1,12$ & & 0,88 & $0,72-1,06$ & \\
\hline Realiza três refeições/dia & & & & & 0,1 & & & 0,09 \\
\hline Nunca / às vezes & 179 & 24,0 & 0,80 & $0,61-1,06$ & & 0,79 & $0,59-1,05$ & \\
\hline Sempre & 401 & 29,9 & 1,00 & - & & 1,00 & - & \\
\hline Consumo diário de frutas/legumes & & & & & 0,8 & & & 0,9 \\
\hline Não & 75 & 29,3 & 0,95 & $0,64-1,41$ & & 0,97 & $0,65-1,44$ & \\
\hline Sim & 492 & 27,9 & 1,00 & - & & 1,00 & - & \\
\hline Consumo diário de carnes & & & & & 0,005 & & & 0,04 \\
\hline Não & 280 & 22,1 & 0,65 & $0,49-0,85$ & & 0,66 & $0,63-1,06$ & \\
\hline Sim & 298 & 34,2 & 1,00 & - & & 1,00 & - & \\
\hline Consumo diário de arroz e feijão & & & & & 0,4 & & & 0,9 \\
\hline Não & 102 & 25,5 & 0,88 & $0,62-1,25$ & & 0,99 & $0,77-1,28$ & \\
\hline Sim & 477 & 28,9 & 1,00 & - & & 1,00 & - & \\
\hline
\end{tabular}

* Teste de Wald.

Paraíba $^{13}$ e da Bahia $^{14}$ e em Fortaleza ${ }^{12}$. Outro aspecto importante a ser considerado é a comparabilidade com estes estudos, devido às diferentes metodologias utilizadas para avaliar o consumo alimentar. Alguns estudos utilizaram como instrumento questionários de frequência alimentar previamente validados ${ }^{14,21,23,26,28}$, enquanto que outros usaram perguntas específicas para avaliar 
o consumo de certos alimentos em diferentes períodos de tempo ${ }^{12,13,22,24,25,27}$. No entanto, todos estes estudos tinham o objetivo de avaliar o consumo habitual e não apenas o consumo atual, o que garante certa comparabilidade entre os resultados. Adicionalmente, todos estes estudos são de base escolar e foram publicados no período 2005-2009, a exceção do estudo de Neutzling et al. ${ }^{26}$, desenvolvido no contexto de uma coorte de nascimentos. De forma similar, foram adotados diversos cuidados metodológicos na execução da presente pesquisa a fim de garantir a qualidade das informações, tais como pré-testes dos instrumentos, estudos piloto, adequação dos instrumentos e treinamento dos entrevistadores.

Os resultados apresentados neste trabalho mostraram um elevado consumo diário de refrigerantes, doces e/ou frituras entre os adolescentes caruaruenses entrevistados, sendo que $60 \%$ referiram o consumo diário de pelo menos um destes produtos e $10 \%$ dos adolescentes informaram consumir os três grupos de alimentos diariamente. Tal cenário é preocupante, tendo em vista que o consumo frequente desses alimentos constitui um importante fator de risco para o desenvolvimento da obesidade e de diversas doenças crônicas não transmissíveis, tais como hipertensão arterial sistêmica, doenças cardiovasculares, vários tipos de câncer, entre outras ${ }^{29}$. Considerando as consequências do consumo destes alimentos para a saúde dos indivíduos, o Ministério da Saúde lançou em 2006 o Guia Alimentar para a População Brasileira, recomendando que o consumo de açúcares e gorduras não ultrapasse uma porção diária ${ }^{8}$.

Dentre os alimentos avaliados, o grupo dos doces apresentou maior consumo diário pelos adolescentes de Caruaru, sendo referidos por 36\% dos entrevistados, seguidos pelos refrigerantes (28\%) e pelas frituras (23\%). Estudo realizado em Pernambuco ${ }^{25}$ avaliou os hábitos alimentares de adolescentes e adultos jovens, e mostrou que o consumo médio de refrigerantes é de 3,8 dias por semana. Por sua vez, outros três estudos realizados na Região Nordeste que avaliaram a prevalência de consumo frequente destes alimentos (quatro ou mais vezes por semana) também encontraram predomínio no consumo de doces (35-49\%) em relação aos refrigerantes (32-35\%) e frituras (20-36\%) $)^{12-14}$. Aplicando-se este mesmo ponto de corte aos nossos resultados, é possível observar prevalências de consumo um pouco maiores para os três grupos de alimentos (63\%, 49\% e 48\%, respectivamente). No entanto, considerando-se apenas o consumo diário destes alimentos, as prevalências observadas em Caruaru foram menores que aquelas encontradas no estado de São Paulo ${ }^{21-23}$, onde o consumo diário de doces esteve entre $62 \%{ }^{22} \mathrm{e}$ $97 \%{ }^{23}$ e aproximadamente a metade dos adolescentes consumiu mais de uma porção de refrigerantes por $\mathrm{dia}^{21}$. Na cidade de Pelotas (RS) o consumo diário de doces e frituras também foi elevado (acima de $50 \%$ para ambos os produtos), enquanto que em Toledo $(\mathrm{PR})^{24}$ e em Niterói $(\mathrm{RJ})^{28}$ as prevalências foram semelhantes aos resultados do presente estudo.

As variações observadas no consumo destes alimentos entre os adolescentes estão de acordo com os resultados da Pesquisa de Orçamento Familiar 2008-20095, a qual mostra diferenças importantes no consumo alimentar segundo as Regiões do Brasil. No estado de Pernambuco, por exemplo, o consumo médio per capita anual é cerca de 18,0 kg para refrigerantes, de 2,0 kg para os alimentos industrializados (ricos em gorduras) e 2,4 kg para o grupo dos doces e produtos de confeitaria. Enquanto no estado de São Paulo, os valores para estes produtos foram $32,0 \mathrm{~kg}, 5,3$ $\mathrm{kg}$ e $3,2 \mathrm{~kg}$, respectivamente. Apesar destes números não representarem o quanto os adolescentes estão consumindo de fato, eles constituem um bom indicador do consumo geral da população, permitindo visualizar diferenças regionais quanto à aquisição destes alimentos, e explicando em parte as variações nas prevalências de consumo entre os estudos.

Embora não tenham sido localizados outros estudos sobre a prevalência de consumo diário destes alimentos em Pernambuco, os resultados observados entre os estudantes de Caruaru são preocupantes, tendo em vista as tendências de consumo na população. Segundo dados da $\mathrm{POF}^{6}$, no período de 2002-03 a 2008-09 este estado apresentou um aumento de $50 \%$ no consumo de doces, $150 \%$ no de alimentos prontos e $13 \%$ para os refrigerantes. Já no estado de São Paulo, o consumo de refrigerantes no mesmo período mantevese estável, e o consumo de doces e alimentos prontos aumentou apenas $14 \%$ e $51 \%$, respectivamente. Adicionalmente, as consequências destas mudanças alimentares no estado nutricional dos adolescentes seguiram o mesmo padrão de aumento. O excesso de peso na Região Nordeste esteve presente em pouco mais de $11 \%$ dos adolescentes em 2003, enquanto que em 2008-09 esse número passou para 16,5\% (aumento de quase 50\%). Em São Paulo, a prevalência de excesso de peso passou de $19 \%$ para $23 \%$ no mesmo período, um aumento de apenas 20\%. 
As presentes análises também mostraram que existem diferenças no consumo de doces conforme o sexo, sendo $25 \%$ maior entre as meninas. Os resultados encontrados no Paraná ${ }^{24}$ corroboram com os achados do nosso estudo, porém na cidade de Pelotas (RS) ${ }^{26,27}$ observou-se um consumo de frituras maior entre os meninos, e o consumo de doces não apresentou diferenças entre os sexos. O estudo de Carmo et al. ${ }^{21}$ em São Paulo também não encontrou diferenças no consumo de doces entre meninos e meninas. Nosso estudo não encontrou nenhuma associação entre sexo e consumo de refrigerantes, concordando com os achados dos estudos realizados em São Paulo ${ }^{21}$, Rio de Janeiro ${ }^{28}$, Florianópolis e Recife $^{25}$ que também não encontraram diferenças. Apenas o estudo realizado no Paraná mostrou maior consumo de refrigerantes entre os meninos. Estas diferenças nos hábitos de consumo dos adolescentes também têm sido evidenciadas para outros alimentos, tais como frutas e vegetais $^{30,31}$, o que aponta para a necessidade de considerar o gênero dos adolescentes nas políticas de saúde que visam mudanças no comportamento alimentar.

O excesso de peso apresentou associação limiar $(\mathrm{p}=0,05)$ com maior prevalência de consumo de doces, mas não com o de refrigerantes ou frituras. Embora este resultado possa ser susceptível à causalidade reversa, um estudo realizado na Paraíba ${ }^{13}$ e outro em São Paulo ${ }^{22}$ encontraram resultados semelhantes, sugerindo que possa existir realmente uma relação direta entre excesso de peso e consumo de doces entre os adolescentes. O consumo de doces foi também maior entre indivíduos que apresentaram consumo diário de arroz e feijão, enquanto que o de frituras foi mais prevalente entre aqueles que consomem carnes diariamente. A comparação destes resultados com outros trabalhos não foi possível, pois não foi encontrado nenhum artigo brasileiro que tenha avaliado esta associação entre adolescentes. No entanto, o consumo de alimentos, sejam estes de proteção ou de risco para a saúde, muitas vezes são consumidos de acordo com os hábitos dos indivíduos, permitindo assim, analisar o comportamento alimentar mediante a avaliação de padrões de consumo ${ }^{32}$.

Embora o número de escolares avaliados pudesse ser considerado como uma possível limitação, considerando o tamanho de amostra disponível e a prevalência dos três desfechos e das diversas variáveis independentes avaliadas, $\mathrm{o}$ presente estudo teve $80 \%$ de poder para detectar $\mathrm{RP}$ entre 1,4 e 1,8 como fatores de risco, bem como valores entre 0,6 e 0,7 como fatores de proteção, para a maioria das variáveis. Apenas para a variável "tabagismo" a amostra teve poder para detectar RP entre 1,9-2,3 como risco ou de 0,4 a 0,5 como proteção. Estas estimativas consideraram um alfa de $5 \%$, assim como uma redução de $10 \%$ no tamanho da amostra, devido ao ajuste para fatores de confusão, e levaram em conta o efeito de delineamento $(1,5)$. Assim, apesar de serem esperadas magnitudes de efeito maiores que as detalhadas acima para grande parte das associações testadas, os desfechos não estiveram associados com a maioria das variáveis demográficas, socioeconômicas e/ou comportamentais, indicando que o elevado consumo de doces, refrigerantes e frituras acontece de forma homogênea entre os adolescentes.

O consumo destes alimentos também foi semelhante entre adolescentes das áreas urbana e rural, o que mostra que as mudanças nos padrões de alimentação com aumento no consumo de alimentos processados não é mais um fenômeno exclusivamente urbano ${ }^{3}$. Esta falta de associação para a maioria das variáveis avaliadas demonstra resultados importantes para a saúde pública, pois as estratégias direcionadas à redução no consumo de alimentos ricos em gordura e/ou açúcares deveriam envolver todos os adolescentes, independentemente do grupo etário, posição socioeconômica e/ou área de residência.

Em conclusão, o estudo encontrou uma elevada prevalência de consumo de doces, refrigerantes e frituras entre adolescentes caruaruenses. Com exceção das diferenças no consumo em algumas características isoladas, foi observado um padrão bastante homogêneo no consumo destes alimentos conforme as distintas variáveis avaliadas. $\mathrm{O}$ monitoramento do consumo destes alimentos entre os adolescentes torna-se fundamental para a elaboração de políticas públicas de curto e longo prazo que visem à formação de hábitos alimentares saudáveis e a redução nas prevalências de DCNT. Recomenda-se que novos estudos sejam realizados para um melhor conhecimento e entendimento das práticas alimentares dos adolescentes, possibilitando, assim, a avaliação das tendências no consumo de alimentos processados. 


\section{Colaboradores}

RV Zanini, LC Muniz, BC Schneider, RM Tassitano, WMN Feitosa e AS González-Chica participaram igualmente de todas as etapas de elaboração do artigo.

\section{Referências}

1. Popkin BM. What can public health nutritionists do to curb the epidemic of nutrition-related noncommunicable disease? Nutrition Reviews 2009; 67(Supl. 1):S79-S82.

2. Øverby NC, Lillegaard ITL, Johansson L, Andersen LF. High intake of added sugar among Norwegian children and adolescents. Public Health Nutrition 2004; 7(2):285-293.

3. Kac G, Sichieri R, Gigante DP, organizadores. Epidemiologia Nutricional. Rio de Janeiro: Ed. Fiocruz, Ed. Atheneu; 2007.

4. Portilho F, Castañeda M, Castro IRR. A alimentação no contexto contemporâneo: consumo, ação política e sustentabilidade. Cien Saude Colet 2011; 16(1):99-106.

5. Instituto Brasileiro de Geografia e Estatística (IBGE). Pesquisa de Orçamentos Familiares 2008-2009: antropometria e estado nutricional de crianças, adolescentes e adultos no Brasil. [página na Internet] 2010. [acessado 2013 set 28]; Disponível em: http://www. ibge.gov.br/home/estatistica/populacao/condicaodevida/pof/2008_2009_encaa/default.shtm

6. Instituto Brasileiro de Geografia e Estatística (IBGE). Pesquisa de Orçamentos Familiares 2002-2003: Análise da Disponibilidade Domiciliar de Alimentos e do Estado Nutricional no Brasil. [página na Internet]. 2004 [acessado 2013 set 28]. Disponível em: http://www.ibge.gov.br/home/estatistica/populacao/condicaodevida/pof/2002analise/default.shtm

7. World Health Organization (WHO). World Helath Report 2002: reducing risks, promoting healthy life. Geneva: WHO; 2002.

8. Brasil. Ministério da Saúde (MS). Secretaria de Atenção à Saúde. Coordenação Geral da Política de Alimentação e Nutrição. Guia Alimentar para a População Brasileira: Promovendo a Alimentação Saudável. Brasília: MS; 2006.

9. Mikkila V, Rasanen L, Raitakari OT, Pietinen P, Viikari J. Longitudinal changes in diet from childhood into adulthood with respect to risk of cardiovascular diseases: The cardiovascular Risk in Young Finns Study. Eur J Clin Nutr 2004; 58(7):1038-1045.

10. Moreno LA, Rodríguez G, Fleta J, Bueno-Lozano M, Lázaro A, Bueno G. Trends of Dietary Habits in Adolescents. Crit Rev Food Sci Nutr 2009; 50(2):106112.

11. Levy RB, Castro IRR, Cardoso LO, Tavares LF, Sardinha LMV, Gomes FS, Costa AWN. Consumo e comportamento alimentar entre adolescentes brasileiros: Pesquisa Nacional de Saúde do Escolar (PeNSE), 2009. Cien Saude Colet 2010; 15(Supl. 2):3085-3097.

12. Silva ARV, Damasceno MMC, Marinho NBP, Almeida LS, Araújo MFM, Almeida PC, Almeida IS. Hábitos alimentares de adolescentes de escolas públicas de Fortaleza, CE, Brasil. Rev Bras Enferm 2009; 62(1):18-24.

13. Nunes MMA, Figueiroa JN, Alves JGB. Excesso de peso, atividade física e hábitos alimentares entre adolescentes de diferentes classes econômicas em Campina Grande (PB). Rev Assoc Med Bras 2007; 53(2):130-134. 
14. Santos JS, Costa MCO, Nascimento-Sobrinho CL, Silva MCM, Souza KEP, Melo BO. Perfil antropométrico e consumo alimentar de adolescentes de Teixeira de Freitas-Bahia. Rev Nutrição 2005; 18(5): 623-632.

15. Nahas MV, Barros MVG, Bem MFLD, Oliveira ESA, Loch MR. Estilo de vida e indicadores de saúde dos jovens catarinenses. Relatório da pesquisa desenvolvida pelo Núcleo de Pesquisa em Atividade Física e Saúde com amostra representativa dos estudantes de 15 a 19 anos matriculados nas escolas estaduais do ensino médio em Santa Catarina. Florianópolis: Universidade Federal de Santa Catarina; 2005.

16. Craig CL, Marshall AL, Sjöström M, Bauman AE, Booth ML, Ainsworth EA. International physical activity questionnaire: 12-Country reliability and validity. Med Sci Sports Exerc 2003; 35(8):1381-1395.

17. Lohman TG, Roche AF, Martorell R. Anthropometric standardization reference manual. Illinois: $\mathrm{Hu}-$ man Kinetics Books; 1988.

18. World Health Organization (WHO). Global database on body mass index: [acessado 2012 jun 16]. Disponível em: http://apps.who.int/bmi/index.jsp

19. World Health Organization (WHO). Multicentre Growth Reference Study Group. WHO child growth standards: length/height-for-age, weight-for-age, weight-for-length, weight-for-height and body mass index-for-age: methods and development. Geneva: WHO; 2006.

20. Barros AJ, Hirakata VN. Alternatives for logistic regression in cross-sectional studies: an empirical camparison of models that directly estimate the prevalence ratio. BMC Med Res Methodol 2003; 3:21.

21. Carmo MB, Toral N, Silva MV, Slater B. Consumo de doces, refrigerantes e bebidas com adição de açúcar entre adolescentes da rede pública de ensino de Piracicaba, São Paulo. Rev Bras Epidemiol 2006; 9(1):121-130.

22. Fagundes ALN, Ribeiro DC, Naspitz L, Garbelini LEB, Vieira JKP, Silva AP, Lima VO, Fagundes DJ, Compri PC, Juliano Y. Prevalência de sobrepeso e obesidade em escolares da região de Parelheiros do município de São Paulo. Rev Paul Pediatr 2008; 26(3):212-217.

23. Toral N, Slater B, Silva MV. Consumo alimentar e excesso de peso de adolescentes de Piracicaba, São Paulo. Revista de Nutrição 2007; 20(5):449-459.

24. Dalla-Costa MC, Júnior LC, Matsuo T. Hábito alimentar de escolares adolescentes de um município do oeste do Paraná. Rev Nutrição 2007; 20(5): 461-471.
25. Nahas MV, Barros MVG, Goldfine BD, Lopes AS, Hallal PC, Farias Júnior JC, Oliveira ES. Physical activity and eating habits in public high schools from different regions in Brazil: the Saude na Boa project. Rev Bras Epidemiol 2009; 12(2):270-277.

26. Neutzling MB, Araújo CLP, Vieira MFA, Hallal PC, Menezes AMB. Frequência de consumo de dietas ricas em gordura e pobres em fibra entre adolescentes. Rev Saude Publica 2007; 41(3):336-342.

27. Neutzling MB, Assunção MCF, Malcon MC, Hallal PC, Menezes AMB. Hábitos alimentares de escolares adolescentes de Pelotas, Brasil. Rev Nutrição 2010; 23(3):379-388.

28. Nogueira FAM, Sichieri R. Associação entre consumo de refrigerantes, sucos e leite, com o índice de massa corporal em escolares da rede pública de Niterói, Rio de Janeiro, Brasil. Cad Saude Publica 2009; 25(12):2715-2724.

29. Ness AR, Maynard M, Frankel S, Smith GD, Frobisher C, Leary SD, Emmett PM, Gunnell D. Diet in childhood and adult cardiovascular and cause mortality: the Boyd Orr cohort. Heart 2005; 91(7):894898.

30. Rasmussen M, Krolner R, Klepp K-I, Lytle L, Brug J, Bere E, Due P. Determinants of fruit and vegetable consumption among children and adolescents: a review of the literature. Part I: quantitative studies. Int J Behav Nutr Phys Act 2006; 3:22.

31. Muniz LC, Zanini RV, Schneider BC, Tassitano RM, Feitosa WMN, González-Chica DA. Prevalência e fatores associados ao consumo de frutas, legumes e verduras entre adolescentes de escolas públicas de Caruaru, PE. Cien Saude Colet 2013; 18(2):393-404.

32. Canuto R, Camey S, Gigante DP, Menezes AMB, Olinto MTA. Focused Principal Component Analysis: a graphical method for exploring dietary patterns. Cad Saude Publica 2010; 26(11):2149-2156.

Artigo apresentado em 29/07/2012

Aprovado em 01/09/2012

Versão final apresentada em 05/09/2012 